\title{
A constitutive model coupling irradiation with two- phase lithiation for lithium-ion battery electrodes
}

\section{Hui Wu, Zhoucan Xie, Yan Wang, Panpan Zhang, Lizhong Sun, Chunsheng Lu \& Zengsheng $\mathrm{Ma}$}

To cite this article: Hui Wu, Zhoucan Xie, Yan Wang, Panpan Zhang, Lizhong Sun, Chunsheng Lu \& Zengsheng Ma (2019): A constitutive model coupling irradiation with two-phase lithiation for lithium-ion battery electrodes, Philosophical Magazine, DOI: 10.1080/14786435.2019.1569767

To link to this article: https://doi.org/10.1080/14786435.2019.1569767

曲 Published online: 23 Jan 2019.

Submit your article to this journal $₫$

山 Article views: 11

View Crossmark data \lceil 


\title{
A constitutive model coupling irradiation with two-phase lithiation for lithium-ion battery electrodes
}

\author{
Hui Wu ${ }^{\mathrm{a} *}$, Zhoucan Xie ${ }^{\mathrm{b} *}$, Yan Wang ${ }^{c}$, Panpan Zhang ${ }^{\mathrm{a}, \mathrm{d}}$, Lizhong Sun ${ }^{\mathrm{a}}$, \\ Chunsheng $\mathrm{Lu}^{\mathrm{d}}$ and Zengsheng $\mathrm{Ma}^{\mathrm{a}}$
}

${ }^{a}$ National-Provincial Laboratory of Special Function Thin Film Materials, and School of Materials Science and Engineering, Xiangtan University, Hunan, People's Republic of China; 'State Key Laboratory of Nonlinear Mechanics, Institute of Mechanics, Chinese Academy of Science, Beijing, People's Republic of China; 'School of Information and Electronic Engineering, Hunan University of Science and Technology, Hunan, People's Republic of China; ${ }^{\mathrm{d} S}$ School of Civil and Mechanical Engineering, Curtin University, Perth, Western Australia, Australia

\begin{abstract}
When lithium-ion batteries serve in extreme environments like space, severe irradiation might induce significant decay of the electrochemical performances and mechanical properties. In this paper, an electrochemical-irradiated constitutive model is proposed to explore the evolution of dislocation, defect and stress in electrodes during a twophase lithiation process. The results from the analytic formulation and finite difference calculations show that, as $\mathrm{Li}$ intercalation proceeding, the hoop stress in the surface of a spherical particle converts from compression into tension because the large lithiation strain and plastic yielding at the front pushes out the material behind it. And the plastic flow resistance continuously increases with increasing irradiation dose result from the impediment of a defect to dislocation glide. There is a clear peak in the distribution of stress at yielding locations due to the competition between dislocations multiplication and defects annihilation. The model is meaningful for thoroughly understanding the micro-mechanism of lithiation deformation and provides a guideline for predicting their mechanical behaviours of lithium-ion batteries in unconventional environment.
\end{abstract}

\section{ARTICLE HISTORY}

Received 16 July 2018

Accepted 27 December 2018

KEYWORDS: Lithium-ion

batteries; two-phase lithiation; irradiation; dislocation

\section{Introduction}

Although they have been widely used as power devices for portable electronics, high performance lithium-ion batteries (LIBs) are still in great need [1]. Electrode materials such as $\mathrm{Si}, \mathrm{Ge}$ and $\mathrm{Sn}$ with extremely a high theoretical specific capacity are a potential substitution of conventional graphite.

CONTACT Yan Wang ywang8@hnust.edu.cn School of Information and Electronic Engineering, Hunan University of Science and Technology, Hunan 411201, People's Republic of China; Zengsheng Ma zsma@xtu. edu.cn $€$ National-Provincial Laboratory of Special Function Thin Film Materials, and School of Materials Science and Engineering, Xiangtan University, Hunan 411105, People's Republic of China

*These authors contributed equally to this paper.

(c) 2019 Informa UK Limited, trading as Taylor \& Francis Group 
However, a serious obstruction to their commercial use is the large volume deformation $(\sim 400 \%$ in full lithiation of $\mathrm{Si})$, resulting in fast capacity loss, short life, instability and even failure [2-11]. Thus, it is necessary to clarify the generation and evolution of diffusion-induced stress during lithiation of electrode materials.

Based on available experiments, plastic deformation appears in high-capacity electrode materials during lithiation [7,9,12-18]. For example, by using a multibeam optical sensor technique, it is shown that the Si thin-film electrode undergoes repeated compressive and tensile-plastic flow during intercalation and deintercalation of $\mathrm{Li}$, and dissipates the mechanical energy [12]. With the help of in situ transmission electron microscopy, a sharp interface $(\sim 1 \mathrm{~nm}$ thickness) was observed between the pristine $\mathrm{Si}$ and lithiated amorphous $\mathrm{Li}_{x} \mathrm{Si}(x=3.75)$ phase during lithiation of single crystal silicon [19], together with a large Li concentration gradient at the two-phase boundary [20]. Such a sharp interface can result in an abrupt change of Li concentrations across the phase boundary, leading to a sudden transformation from compression to tension of diffusioninduced stress [21].

Recently, several trans-scale theoretical models were also established to describe the deformation behaviour of electrode materials during charging and discharging [22-24]. For instance, an elasto-plastic non-linear two-phase lithiation model was proposed to obtain the dynamic evolution of Li-ion concentration and diffusion-induced stress [22,23,25]. Besides, the plasticity origin of Si electrodes was simulated by first-principles calculation [26]. A framework of mechanics was presented for a physical understanding of stress generation during lithiation of high-capacity electrode materials [27]. Zuo et al. built up a phase field model for lithium diffusion, finite deformation, stress evolution and crack propagation in LIBs [27]. The effects of particle shape and concurrent plasticity on stress generation during lithiation were studied in particulate LIB electrodes. These models can well explain diffusion-induced stress and fracture behaviours due to the electrochemical reaction under regular environments.

During deep space exploration, however, LIBs suffer severe irradiation effects, high radiation field and post-nuclear accident scenario [28-32]. Qiu et al. [33] and Samin et al. [34] characterised the surface morphologies of cathode materials before and after neutron and gamma-ray irradiation with a growing particle size and reported that irradiation causes disordering of crystal structures in a post-irradiation sample, which further results in capacity loss of batteries. Deterioration was detected because of the irradiation effect on electrode and electrolyte materials [32]. Recently, Ma et al. [35] developed an electrochemical-irradiated plasticity model to study the effects of irradiation and diffusion-induced stress on the mechanical properties of cylindrical electrodes, and showed that the yield strength of irradiated electrode materials increases with dose. The influence of irradiation on electrodes is due to the 
change of microstructures [36-41]. For instance, a typical irradiation hardening phenomenon was observed in materials with a body-centered cubic structure $(\mathrm{Cu})$ [42-44] and a face-centered cubic structure (Fe) [45-48]. A quantitative in situ nano-compression test was conducted to study the evolution of defect and dislocation of ion beam irradiated materials [49]. The electrochemical reaction dislocations in a single nanowire were continuously nucleated and absorbed at the moving reaction front [14], and irradiation defects were observed [50].

Unlike the models with only consideration of dislocation interaction (e.g. the Kocks-Mecking model) [51,52], the irradiation hardening model has been developed and used to describe the interaction between defect and dislocation $[43,53]$. Here, microscopic irradiation-induced defects and dislocations were introduced into a constitutive model that couples the irradiation hardening and two-phase interface in a nano-spherical particle [22,54-58].

In this paper, to explore the evolution of dislocations, defects and stress associated with a two-phase lithiation boundary, a constitutive model coupling irradiation with electrochemical reaction is established, which mainly involves the interaction between the electrochemical reaction dislocations and irradiation defects in a cathode particle of LIBs. The paper is organised as follows. Section 2 represents a theoretical framework that takes into account the general elastoplastic lithiation deformation of associated with two-phase boundary and the plastic correction based on an irradiation hardening model. Then, in Section 3 , numerical results of the evolution of defects, dislocation density and stress are analysed. Finally, conclusions are given in Section 4.

\section{Theoretical framework}

\subsection{A general mechanical constitutive model}

Since a large volume swelling due to insertion of Li-ions is accommodated by the elasto-plastic deformation in lithiated electrode particles, the total strain rate $\dot{\varepsilon}_{i j}$ can be written as

$$
\dot{\varepsilon}_{i j}=\dot{\varepsilon}_{i j}^{e}+\dot{\varepsilon}_{i j}^{p}+\dot{\varepsilon}_{i j}^{c} \text {, }
$$

where $\dot{\varepsilon}_{i j}^{e}$ and $\dot{\varepsilon}_{i j}^{p}$ are elastic and plastic strain rates, respectively. $\dot{\varepsilon}_{i j}^{c}$ is the chemical strain rate caused by $\mathrm{Li}$ insertion that is proportional to the rate of normalised $\mathrm{Li}$ concentration $\dot{\mathcal{c}}$, that is

$$
\dot{\varepsilon}_{i j}^{c}=\beta \dot{c} \delta_{i j}
$$

where $\beta=\left(\Omega^{\text {lithiated }} / \Omega^{\mathrm{SnO}_{2}}\right)$ is a volume expansion coefficient of electrode particles, $\Omega^{\mathrm{SnO}_{2}}$ is the volume of $\mathrm{SnO}_{2}$ atom, and $\Omega^{\text {lithiated }}$ represents the volume of lithiated part. $c$ ranging from 0 to 1 is the Li-ion concentration normalised at the maximum Li-ion concentration $c_{0}$ (the full lithium state). $\delta_{i j}$ is the 
Kronecker delta. According to Hooke's law, the elastic strain rate $\dot{\varepsilon}_{i j}^{e}$ can be represented as

$$
\dot{\varepsilon}_{i j}^{e}=\frac{1}{E}\left[(1+v) \dot{\sigma}_{i j}-v \dot{\sigma}_{k k} \delta_{i j}\right],
$$

where $E$ and $v$ are the elastic modulus and Poisson's radio. The plastic strain rate $\dot{\varepsilon}_{i j}^{p}$ complying with the $J_{2}$-flow theory of plasticity is proportion to the deviatoric stress $\sigma_{i j}^{\prime}=\sigma_{i j}-\sigma_{k k} \delta_{i j} / 3$, namely

$$
\dot{\varepsilon}_{i j}^{p}=\frac{3 \dot{\varepsilon}^{p}}{2 \sigma_{e}} \sigma_{i j}^{\prime} .
$$

As the von Mises effective stress $\sigma_{e}=\sqrt{3 \sigma^{\prime}{ }_{i j} \sigma^{\prime}{ }_{i j} / 2}$ is equal to the yield stress, $\dot{\varepsilon}^{p}=\sqrt{(2 / 3) \dot{\varepsilon}_{i j}^{p} \dot{\varepsilon}_{i j}^{p}}$ refers to the plastic strain rate.

\subsection{Yield function}

In irradiation environments, the mechanical properties of materials are affected by the coupling of dislocation and defect. Based on previous studies [20,59], the slip shear stress $\tau_{y}$ is associated with dislocations and defect densities. For a single crystal, a rate-independent yield function, $F\left(\sigma, \tau_{y}\right)[14,26,28-32,36,60]$ can be written as

$$
F\left(\sigma, \tau_{y}\right)=\sigma_{e}-\tau_{y}
$$

where the total shear strain $\tau_{y}$ on a slip plane is given in terms of the total microstructures density $\rho_{t}$ by Franciosi and Zaoui [61]

$$
\tau_{y}=\eta G b \sqrt{\sum_{t}^{N} K_{t} \rho_{t}}=\eta G b \sqrt{K \rho_{d}+L \rho_{f}}
$$

where $\eta$ is a statistical parameter [62], $G$ is the shear modulus, $b$ is Burger's vector, $K_{t}$ is an interaction coefficient of microstructures, and $\rho_{d}$ is the density of dislocations. The density of defect $\rho_{f}$ can be calculated by

$$
\rho_{f}=N_{f} d_{f}
$$

Correspondingly, $K$ is the dislocation coefficient related to the slip systems and $L$ is the defect coefficient representing the interaction between dislocations and defects [63]. $N_{f}$ is the number density and $d_{f}$ is the average size of stacking fault tetrahedrons (SFTs). Thus, $F\left(\sigma, \tau_{y}\right)$ can be expressed as

$$
F\left(\sigma, \tau_{y}\right)=\sigma_{e}-\eta G b \sqrt{K \rho_{d}+L \rho_{f}} .
$$

The plastic deformation of a metal is realised through the dislocation slip inside grains. The generation of dislocations is based on the resistance to dislocation 
movement posed by forest dislocations acting as random obstacles. That is, the expansion of dislocation loops origins from the Frank-Read type sources. The mutual annihilation between parallel dislocations with the same character but the opposite sign is assumed to be the predominant annihilation mechanism. Thus, the evolution of dislocations is controlled by the growth and dynamic annihilated terms, namely,

$$
\dot{\rho}_{d}=\frac{1}{b}\left(\frac{1}{S}-2 \rho_{d} y_{c}\right)\left|\dot{\varepsilon}^{p}\right|,
$$

where $y_{c}$ is the critical edge annihilation distance, and $S$ represents the mean free path of dislocation segment that is related to the dislocation density,

$$
S=\xi \rho_{d}^{-(1 / 2)}
$$

where $\xi$ is a dimensionless proportionality constant controlling the mobility of dislocations.

According to molecular dynamics studies, the interaction of different dislocations with SFTs is rather complicated [64-67]. The defect clusters such as SFTs are annihilated by active dislocations or shrunk to a smaller size. The interaction between dislocations and defects may lead to a possible annihilation when SFTs are overlapped or truncated $\sim 70-80 \%$ of defects [63]. As shown in Figure 1, the video-frame images exhibit the interaction between a truncated SFT and a dislocation, indicating that SFT2 was completely annihilated by moving dislocations [50]. Based on the schematic diagram of annihilation (see Figure 1), dislocations glide and then interact with SFTs on the glide plane, which could make the defect split up and transform into other types,
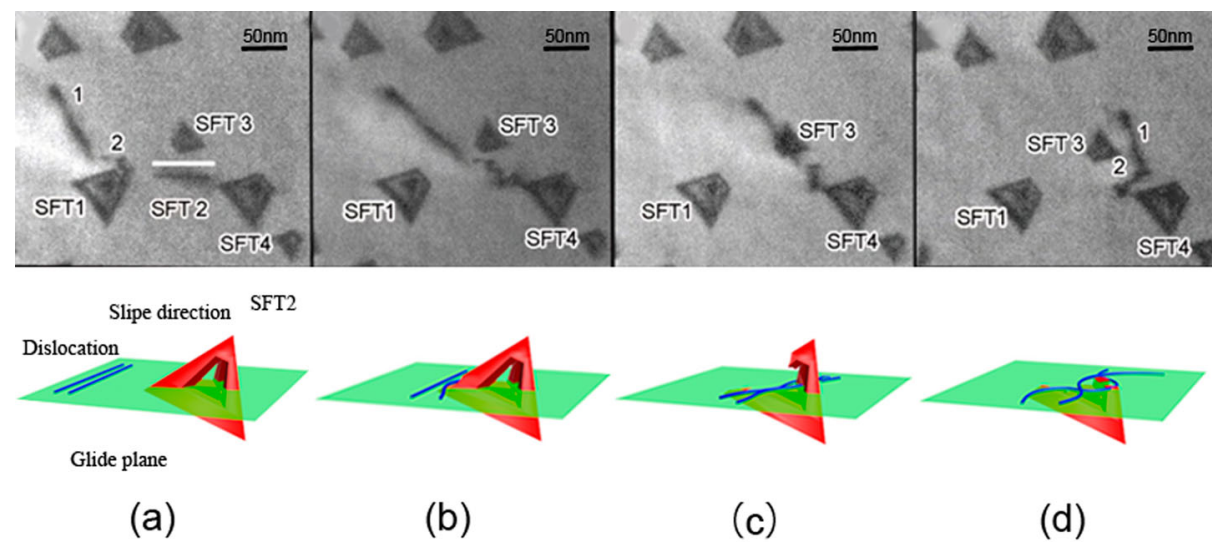

Figure 1. Annihilation mechanism of defects: video-frame images showing the interaction between a truncated SFT and a dislocation, where SFT2 was completely annihilated by a moving dislocation. Snapshots were acquired at (a) $0 \mathrm{~s}$, (b) $1.53 \mathrm{~s}$, (c) $2.03 \mathrm{~s}, 3.97$ and (d) $15.54 \mathrm{~s}$ (photographs are adapted from [50]); and the schematic diagram of interaction between dislocation and defect corresponding to the experiment upon. 
such as a dislocation loop, and eventually being annihilated. The rate of defect annihilation $\dot{\rho}_{f}$ can be written as

$$
\dot{\rho}_{f}=-A_{s} \rho_{d}\left(\rho_{f}-\rho_{f s}\right) \frac{d_{f}}{b}\left|\dot{\varepsilon}^{p}\right|,
$$

where $A_{\mathrm{s}}$ is the effective area of defect annihilation (see Figure 2), and

$$
A_{s}=2 d_{s} S+\pi d_{s}^{2}
$$

where a dislocation moves with the standoff distance $d_{s}$ and $S$ is the moving distance of a dislocation before it meets a defect in $\mathrm{d} t . \rho_{f \mathrm{~s}}$ is the steady-state value of defect density. In Equation (11), when $\rho_{f}-\rho_{f s}=0$, the rate of defect annihilation is zero.

\subsection{Elasto-plastic deformation during lithiation}

In order to study the microstructural evolution during lithiation with irradiation, it is necessary to consider the migration of phase boundaries. Thus, a series of one-dimensional step-like profiles of Li-ion distribution with the flexible sigmoid function are assumed to simulate the movement of a sharp boundary between unlithiation and lithiation phases $[26,68]$, viz.

$$
\frac{c(r, t)}{c_{0}}=\frac{1}{1+\exp [-\gamma(r-(R-k t) / R)]},
$$

where $c(r, t)$ is the Li concentration at the location $r$ and time $t$. $c_{0}$ is the concentration at a steady stage. Physically, the lithiation involves two processes: Li diffusion through the lithiated phase and the chemical reaction at the twophase boundary. $\gamma=D / k R$ is the dimensionless parameters representing the relative rate of diffusion and reaction with a sharp profile of Li concentration jumping from 0 to 1 , where $D$ is the diffusivity of $\mathrm{Li}$ in a Li-rich phase, $k$ is the rate at the front of reaction, and $R$ is the particle size. The larger $\gamma$, the faster Li diffuses, implying that lithiation is limited by electrochemical reaction.

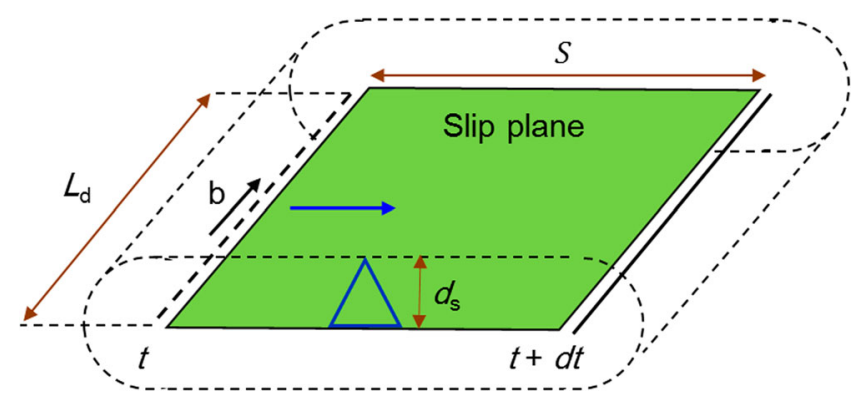

Figure 2. Schematic of dislocation glide with the standoff distance $d_{\mathrm{s}}$ between dislocations and SFTs, and the mean free path $S$ of dislocations. Dislocations are expressed by solid lines and SFTs are shown as triangles. 
It is important for two-phase lithiation to consider the effect of irradiateddefects evolution and stress generation on the rate at the reaction front. Some works have shown that the defects can make the initial Li insertion process thermodynamically more favourable [27]. Kim and Qi [69] described that the lithiation rate exhibits an exponential relationship with the vacancy concentration,

$$
k \propto \exp \left[\frac{c_{v} * E_{f}}{k_{B} T}\right],
$$

where $c_{v}$ is the defect concentration at the two-phase boundary, $E_{f}$ the defect formation energy, $k_{B}$ the Boltzmann constant, and $T$ the temperature.

On the other hand, the stress fields across the reaction front act as an energy barrier for lithiation reaction and hinder the movement of the reaction front [26]. The stress-induced energy barrier for lithiation reaction is given by

$$
\Delta G=\Delta G_{r}-e \Phi+\Delta G_{\text {stress }}=\Delta G_{r}-e \Phi-\Omega \sigma_{m} .
$$

where $\Delta G$ is the free energy of the lithiation reaction when both the stress and voltage are ignored. In the sign convention, a negative $\Delta G$ drives lithiation, and a negative value represents a larger driving force. The voltage contributes to the work $e \Phi$, where $\Phi$ and $e$ are the voltage and elementary charge, respectively. When one lithium atom is incorporated into lithiated materials, the mean stress, $\sigma_{\mathrm{m}}$, does the work $\Omega \sigma_{\mathrm{m}}$, where $\Omega$ is the volume change caused by insertion of lithium atoms.

Thus, the reaction is taken to be thermally-activated by the kinetic model [26]

$$
k=k_{0} * \exp \left[\frac{c_{v} * E_{f}}{k_{B} T}\right] \exp \left[-\frac{\Delta G_{\mathrm{r}}-\sigma_{\mathrm{m}} \Omega}{k_{B} T}\right] .
$$

here, $k_{0}$ is a parameter analogous to the exchange current density in a redox process.

In a spherical coordinate system $(r, \theta, \varphi)$ in Figure 3, it is assumed that $\dot{\varepsilon}_{\theta}=\dot{\varepsilon}_{\varphi}$, and the strain component can be written as

$$
\begin{gathered}
\dot{\varepsilon}_{r}=\dot{\varepsilon}_{r}^{e}+\dot{\varepsilon}_{r}^{p}+\dot{\varepsilon}_{r}^{c} \\
\dot{\varepsilon}_{\theta}=\dot{\varepsilon}_{\varphi}=\dot{\varepsilon}_{\theta}^{e}+\dot{\varepsilon}_{\theta}^{p}+\dot{\varepsilon}_{\theta}^{c}
\end{gathered} .
$$

Therefore, based on Equation (2), the chemical strain can be simplified as

$$
\dot{\varepsilon}_{r}^{c}=\dot{\varepsilon}_{\theta}^{c}=\dot{\varepsilon}_{\varphi}^{c}=\beta \dot{c} .
$$

Similarly, the elastic strain rate of Equation (3) is described as

$$
\begin{gathered}
\dot{\sigma}_{r}=\frac{E}{(1-v)(1-2 v)}\left[(1-v) \dot{\varepsilon}_{r}^{e}+2 v \dot{\varepsilon}_{\theta}^{e}\right] \\
\dot{\sigma}_{\theta}=\dot{\sigma}_{\varphi}=\frac{E}{(1-v)(1-2 v)}\left[\dot{\varepsilon}_{\theta}^{e}+v \dot{\varepsilon}_{r}^{e}\right]
\end{gathered}
$$



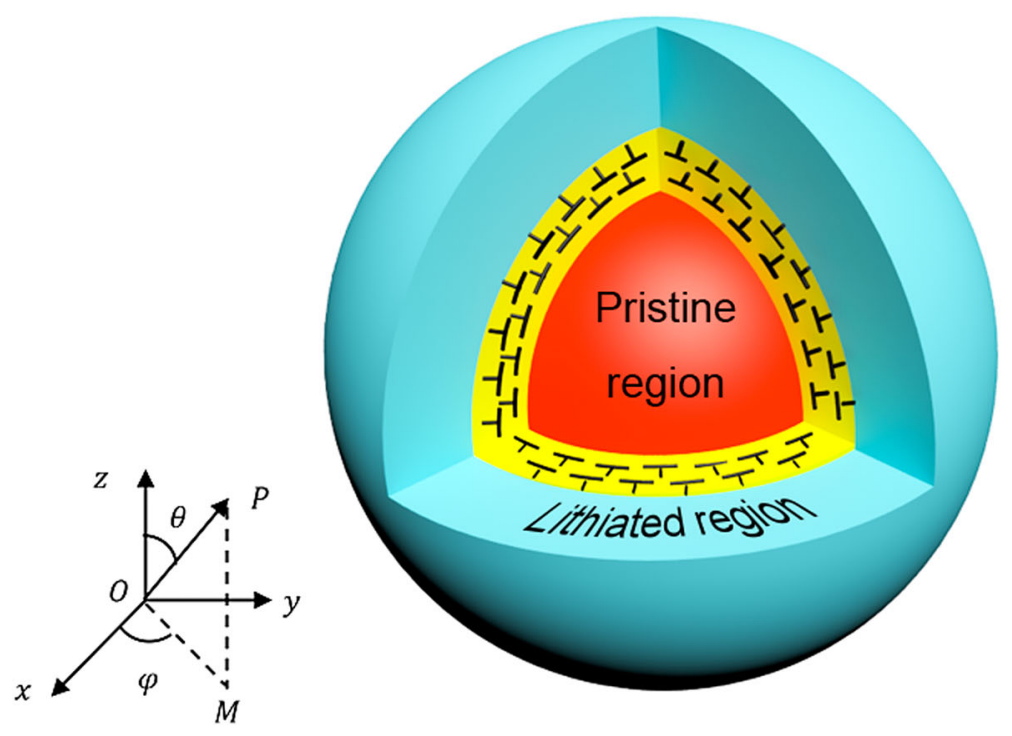

Element $\mathrm{A}$ $(r=0.9 R)$ Dislocation

Figure 3. Schematic illustration of the two-phase mechanism for Li-ion diffusion and the element A located in $r / R=0.9$.

The plastic strain rate of Equation (4) can be written as

$$
\begin{gathered}
\dot{\varepsilon}_{r}^{p}=\dot{\bar{\varepsilon}}^{p} \frac{3 \sigma_{r}^{\prime}}{2 \sigma_{e}} \\
\dot{\varepsilon}_{\theta}^{p}=\dot{\varepsilon}_{\varphi}^{p}=\dot{\bar{\varepsilon}}^{p} \frac{3 \sigma_{\theta}^{\prime}}{2 \sigma_{e}}
\end{gathered}
$$

and the deviatoric stresses are resolved by

$$
\begin{gathered}
\sigma_{r}^{\prime}=\frac{1}{3}\left(\sigma_{r}-\sigma_{\theta}\right) \\
\sigma_{\theta}^{\prime}=\sigma_{\varphi}^{\prime}=\frac{1}{3}\left(\sigma_{\theta}-\sigma_{r}\right)
\end{gathered}
$$

where the von Mises effective stress is

$$
\sigma_{e}=\sqrt{\frac{3}{2} \sigma_{i j}^{\prime} \sigma_{i j}^{\prime}}=\left|\sigma_{r}-\sigma_{\theta}\right|
$$

To control the computation stabilisation with a sharp concentration boundary, the rate-dependence must have an asymptotic value. Thus, a power-exponential function was developed to express the equation the equivalent plastic strain [70,71], that is

$$
\dot{\bar{\varepsilon}}^{p}=\dot{\varepsilon}_{\mathrm{eq}}^{p}\left(\frac{\sigma_{e}}{\sigma_{\text {flow }}}-1\right)^{\frac{1}{m}}\left(\frac{1}{1+\exp \left[-80\left(\left(\sigma_{e} / \sigma_{\text {flow }}\right)-1\right)\right]}\right),
$$

where $\dot{\varepsilon}_{\mathrm{eq}}^{p}=\sqrt{(2 / 3) \dot{\varepsilon}_{i j}^{p} \dot{\varepsilon}_{i j}^{p}}$ represents the equivalent plastic strain rate, the plastic flow resistance $\sigma_{\text {flow }}$ is equivalent to the function of $\tau_{y}$ as given by Equation (8), and $m$ is 
the rate-dependent sensitivity exponent. For a spherically-symmetric problem, the equilibrium equation is given by

$$
\frac{d \dot{\sigma}_{r}}{d r}+\frac{2}{r}\left(\dot{\sigma}_{r}-\dot{\sigma}_{\theta}\right)=0
$$

Using deformation rate $V_{r}$ to express the strain rate, we have

$$
\begin{gathered}
\dot{\varepsilon}_{r}=\frac{d V_{r}}{d r} \\
\dot{\varepsilon}_{\theta}=\dot{\varepsilon}_{\varphi}=\frac{V_{r}}{r} .
\end{gathered}
$$

There is zero stress boundary in the surface of the particle and zero velocity of deformation along the radial with the radius $R$, namely

$$
\begin{aligned}
& \dot{\sigma}_{r}(r=R)=0 \\
& V_{r}(r=0)=0
\end{aligned},
$$

Based on the analytic formulation above, the stress rate can be obtained by Equations (17), (19) and (25), that is

$$
\begin{gathered}
\dot{\sigma}_{r}=\frac{E}{(1+v)(1-2 v)}\left[(1-v)\left(\frac{d V_{r}}{d r}-\dot{\varepsilon}_{r}^{c}-\dot{\varepsilon}_{r}^{p}\right)+2 v\left(\frac{V_{r}}{r}-\dot{\varepsilon}_{\theta}^{c}-\dot{\varepsilon}_{\theta}^{p}\right)\right] \\
\dot{\sigma}_{r}-\dot{\sigma}_{\theta}=\frac{E}{(1+v)}\left[\frac{d V_{r}}{d r}-\frac{V_{r}}{r}-\dot{\varepsilon}_{r}^{c}-\dot{\varepsilon}_{r}^{p}+\dot{\varepsilon}_{\theta}^{c}+\dot{\varepsilon}_{\theta}^{p}\right]
\end{gathered}
$$

\subsection{Finite difference method}

Combining Equations (9) and (10), the rate dislocation and defect densities can be written as,

$$
\begin{gathered}
\frac{d \rho_{d}}{d t}=\frac{1}{b}\left(\frac{1}{\kappa\left(\rho_{d}\right)^{1 / 2}}-2 \rho_{d} y_{c}\right)\left|\dot{\varepsilon}^{p}\right|, \\
\frac{d \rho_{f}}{d t}=-A_{s}\left(\rho_{f}-\rho_{f_{s}}\right) \frac{d_{f}}{b}\left|\dot{\varepsilon}^{p}\right| .
\end{gathered}
$$

For given plastic strain rates at time $t$, Equations (29) and (30) become firstorder ordinary differential equations to gain the evolution of dislocation and defect by the finite difference and iterative shooting methods. Thus, Equations (29) and (30) can be rewritten as

$$
\begin{gathered}
\frac{d \rho_{d}}{d t}=\frac{\rho_{d}^{(i)}-\rho_{d}^{(i-1)}}{\Delta t}, \\
\frac{d \rho_{\mathrm{def}}}{d t}=\frac{\rho_{\mathrm{def}}^{(i)}-\rho_{\mathrm{def}}^{(i-1)}}{\Delta t} .
\end{gathered}
$$


The next node is calculated by iterating. In addition to, the initial defect density $\rho_{f 0}$ in Table 1 is used to represent the irradiation degree and the initial value of dislocation is $2.0 \times 10^{-6} \mathrm{~nm}^{-2}$ in Table 2 [62]. Similarly, substituting Equations (27) and (28) into Equation (24), the equilibrium equation can be expressed by $V_{r}$, that is

$$
\begin{aligned}
\frac{d^{2} V_{r}}{d r^{2}}+\frac{2}{r} \frac{d V_{r}}{d r}-\frac{2 V_{r}}{r^{2}}= & \frac{1}{1-v} \frac{d}{d r}\left[(1-v)\left(\dot{\varepsilon}_{r}^{c}+\dot{\varepsilon}_{r}^{p}\right)+2 v\left(\dot{\varepsilon}_{\theta}^{c}+\dot{\varepsilon}_{\theta}^{p}\right)\right] \\
& +\frac{2(1-2 v)}{1-v} \frac{1}{r}\left[\left(\dot{\varepsilon}_{r}^{c}+\dot{\varepsilon}_{r}^{p}\right)-\left(\dot{\varepsilon}_{\theta}^{c}+\dot{\varepsilon}_{\theta}^{p}\right)\right] .
\end{aligned}
$$

Once chemical and plastic strain rates are obtained, Equation (33) becomes a second-order ordinary differential equation of $V_{\mathrm{r}}$. Specifically, the radial distance of a spherical particle is discretized into $n$ points $r^{(i)} \in[0, R],(i=1, \ldots$, $n)$, with an equal spacing $\Delta r=R /(n-1)$, and then Equation (33) is discretized by

$$
\frac{d^{2} V_{r}}{d r^{2}}=\frac{V_{r}^{(i+1)}+V_{r}^{(i-1)}-2 V_{r}^{(i)}}{\Delta r^{2}}, \frac{d V_{r}}{d r}=\frac{V_{r}^{(i+1)}+V_{r}^{(i-1)}}{2 \Delta r},
$$

where $V_{r}^{i}$ is the radial velocity at $r^{(i)}$. To determine $V_{r}^{i}$, we can start from $V_{r}^{1}\left(r^{1}=0\right)=0$ by the boundary condition of Equation (27), make an initial guess of $V_{r}^{2}$ and solve $V_{r}^{3} \ldots V_{r}^{n}$ based on Equations (33) and (34), and then calculate $\dot{\sigma}_{r}(r=R)$ according to Equation (27). Due to the traction-free boundary condition of Equation (26), the above calculations are repeated to find out an optimal $V_{r}^{2}$, making $\dot{\sigma}_{r}(r=R)$ approach to zero to update $V_{r}^{i}$ at time $t$. Hence, stress rates at time $t$ are calculated based on Equations (27) and (28), and then the plastic strain rates are updated at time $t+\Delta t$ based on Equations (20-23). For a given chemical strain rate at time $t+\Delta t, \dot{c}$ at $t+\Delta t$ is calculated based on a prescribed time step. Further, the above steps can be repeated to determine $V_{r}^{i}$ at $t+\Delta t$, accomplishing a loop of numerical integration.

\section{Results and discussion}

As shown in Figure 4(a), irradiation causes the yield stress increase of metals such as $\mathrm{Cu}, \mathrm{Fe}$ and Sn. In Figure 4(b), the yield stress increases with neutrons fluence based on the Hall-patch relationship, that is

$$
\sigma_{Y}=\sigma_{0}+a d_{g}^{-0.5}
$$

Table 1. Initial defect density and its stabilised value of different radiation conditions [63].

\begin{tabular}{lc}
\hline$\rho_{f 0}\left(\mathrm{~nm}^{-2}\right)$ & $\rho_{f s}\left(\mathrm{~nm}^{-2}\right)$ \\
\hline 0 & 0 \\
$1.0 \times 10^{-5}$ & $3.0 \times 10^{-6}$ \\
$1.0 \times 10^{-4}$ & $3.0 \times 10^{-5}$ \\
$5.0 \times 10^{-4}$ & $1.5 \times 10^{-5}$ \\
\hline
\end{tabular}


Table 2. Model parameters and their detail illustrations for high capacity electrode materials.

\begin{tabular}{lcccc}
\hline Parameter & Definition & Value & Unit & Reference \\
\hline$v$ & Poisson's ratio & 0.3 & - & {$[63]$} \\
$E_{0}$ & Elastic modulus & 51.41 & $\mathrm{GPa}$ & \\
$m$ & Strain rate sensitivity & 0.01 & - & {$[72]$} \\
$b$ & Burgers vector & 0.25 & $\mathrm{~nm}$ & {$[40]$} \\
$\beta$ & Lithiation expansion coefficient & 0.26 & - & {$[27]$} \\
$\mathrm{K}$ & Empirical coefficient & 1.15 & - & {$[63]$} \\
$\mathrm{L}$ & Empirical coefficient & 1.47 & - & \\
$\eta$ & Statistical parameter & 32 & & \\
$\rho_{\mathrm{d}}$ & Initial dislocation density & $2.0 \times 10^{-6}$ & $\mathrm{~nm}^{-2}$ & {$[62]$} \\
$\xi$ & Empirical constant & 71.43 & - & \\
$y_{c}$ & Annihilation radius & 1 & $\mathrm{~nm}$ & {$[73]$} \\
$d_{\text {def }}$ & Critical edge annihilation distance & $2.5( \pm 0.5)$ & $\mathrm{nm}$ & {$[63]$} \\
$d_{s}$ & Standoff distance & $1 \sim 3$ & $\mathrm{~nm}$ & \\
$\dot{\bar{\varepsilon}}_{0}^{p}$ & The efficient strain rate constant & 0.001 & -- & {$[74]$} \\
$E_{f}$ & The defect formation energy & 0.23 & $\mathrm{eV}$ & {$[75]$} \\
\hline
\end{tabular}

where $\sigma_{Y}$ is the yield stress, $\sigma_{0}$ is the lattice frictional stress, $a$ is a constant, and $d_{g}$ is the grain size. Due to the existence of a large number of irradiated defects, the movement of internal dislocations will be hindered by defects (called irradiation hardening) when the material is subjected to plastic deformation under external loading. For a given strain increment of $d_{\varepsilon}=3.0 \times 10^{-6}$, Figure 5 shows the stress-strain curves at different irradiation doses. At the elastic stage, there is almost no influence of irradiation on the stress-strain relations of electrodes, however, the mechanical properties in the plastic stage are completely different: (1) after irradiation, the yield stress rises with increasing the doses, which can be attributed to irradiation hardening because defects impede the dislocation slippage; (2) when irradiation doses exceed a certain value, such as $\rho_{f 0}=1.0 \times 10^{-4} \mathrm{~nm}^{-2}$, the flow stress increases initially, and then it drops at the upper yield point. The evolution of flow stress after the yield point is controlled by the competitive mechanism of defect annihilation and dislocation multiplication [40]. On the one hand, the flow stress might decrease because dislocations are unlocked and free to move resulting from the defect annihilation with the
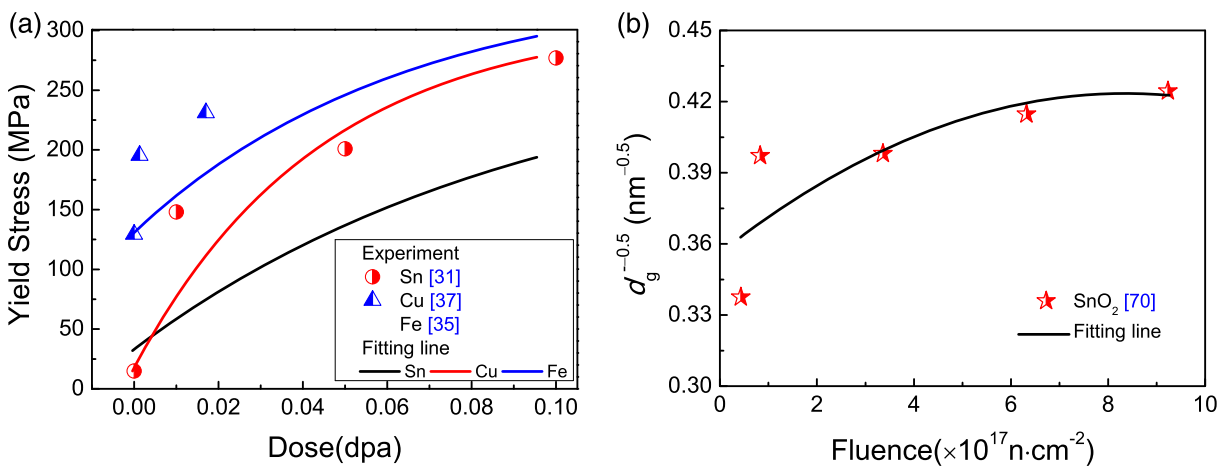

Figure 4. (a) The influence of irradiation dose on the yield stress of $\mathrm{Cu}, \mathrm{Fe}$ and $\mathrm{Sn}$ and (b) the evolution of $d_{\mathrm{S}}^{-0.5}$ for $\mathrm{SnO}_{2}$ film as a function of neutron fluence. 


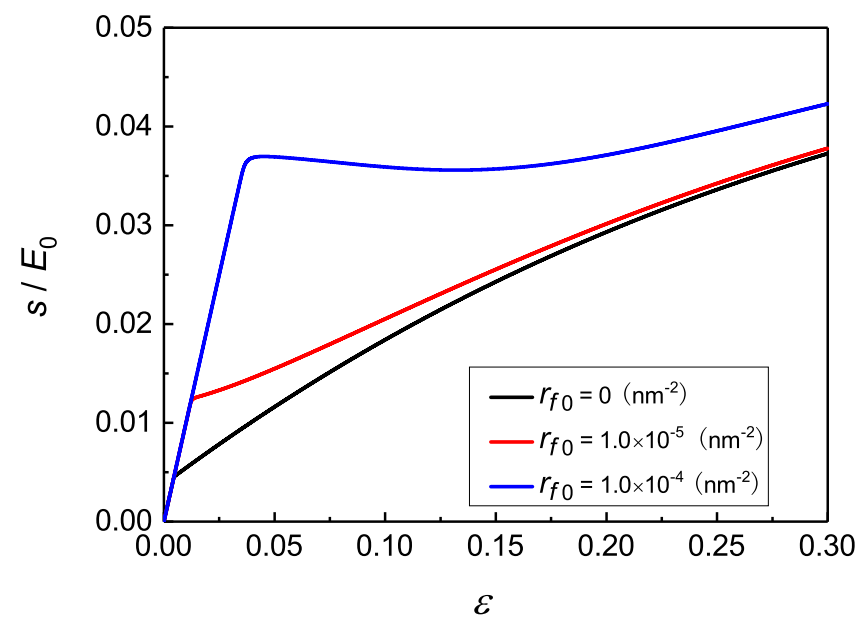

Figure 5. The constitutive relation of uniaxial tension using a constitutive model with different irradiation intensities.

increase of load. On the other hand, the flow stress could increase because of dislocation multiplication. When irradiation dose is large enough, the influence of defect annihilation on flow stress is more than that of dislocation multiplication at the initial phase. Therefore, the flow stress decreases initially due to high defect annihilation but rises with increasing dislocation multiplication.

As shown in Figure 6(a), the $\mathrm{Li}$ concentration distribution under three different lithiated states exhibits an obvious feature of sharp concentration transition which separates the Li-poor and Li-rich phase. The corresponding contours show a two-phase boundary moving to the particle centre with increasing lithiation time in Figure 6(b).

The densities of defects and dislocations are affected by irradiation and their interaction in Figure 7. In Figures $7(\mathrm{a}-\mathrm{c})$, at $k t / R=0.1$, a high-density dislocation appears in a two-phase boundary because the sharp concentration leads to a large misfit strain and dislocations spontaneously nucleate at the interface. Interaction of defects and slip dislocations results in translation of defect type, and further leads to defect annihilation. Thus, near the particle surface, the defect density suddenly decreases. When the initial defect density is $1.0 \times 10^{-5} \mathrm{~nm}^{-2}$, the dislocation density is higher than the defect density in the lithiated region; On the contrary, when the initial defect densities reach to $1.0 \times 10^{-4} \mathrm{~nm}^{-2}$ and $5.0 \times 10^{-4} \mathrm{~nm}^{-2}$, the dislocation densities are smaller than that of defects. When the abrupt boundary moves to the particle centre at $k t / R=0.5$, as shown in Figures $7(\mathrm{~d}-\mathrm{e})$ the sharp density boundary has moved toward the centre corresponding to the moving of reaction front. And the dislocation density is still greater than the defect density in the lithiated region. Nevertheless, in Figure 7(f), for $\rho_{f 0}=5.0 \times 10^{-4} \mathrm{~nm}^{-2}$, the dislocation density is much lower than the defect density, which limits the degree of dislocation multiplication. Here, it is worth noting that there exists a turning point around $1.0 \times 10^{-4} \mathrm{~nm}^{-2}$ in Figures $7(\mathrm{~b})$ and (e), where the densities 

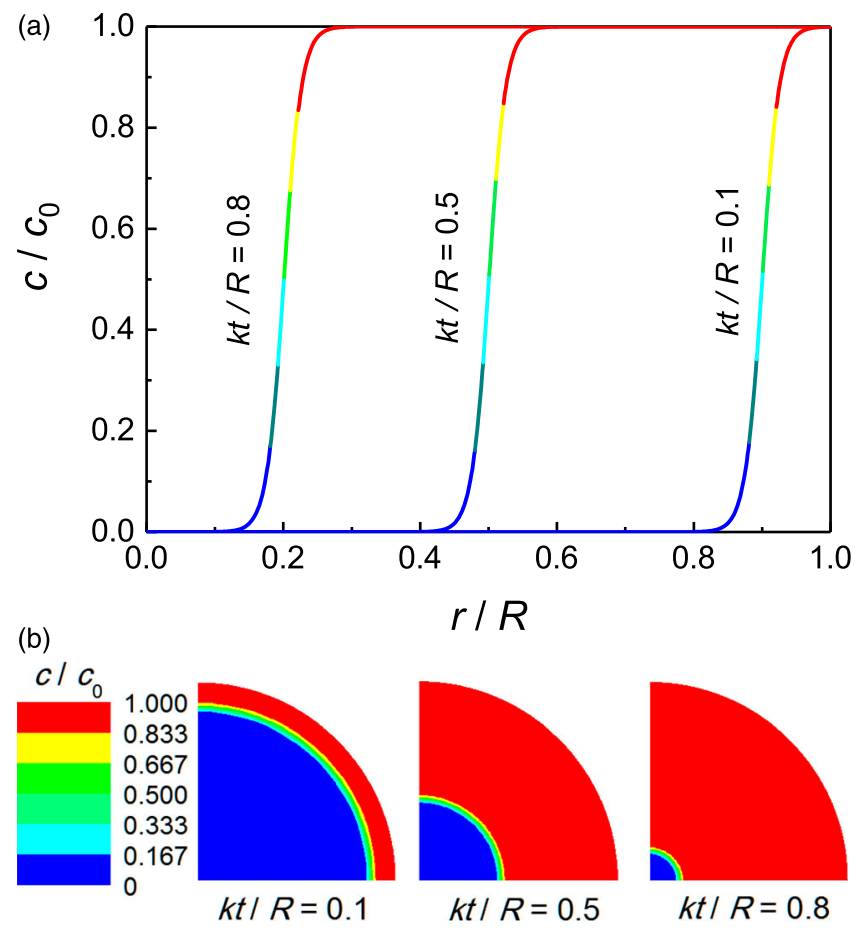

Figure 6. Radial distributions of Li concentration under different lithiated states: (a) the axial concentration graph and (b) the corresponding contour.

of defects and dislocations have an asymptotical phenomenon, reflecting the effect of irradiation on the evolution of microstructures and the fact that the dislocation will be continuously nucleated and absorbed by interface during the reaction.

As shown in Figures 8(a-c), the dislocation density of element A (see Figure 3) rises abruptly with lithiation process, indicating that dislocations readily nucleate at the two-phase boundary and induce local plastic. Finally, the dislocations are absorbed by the moving interaction interface and reached to a stable value. On the other hand, with the increase of irradiation dose, dislocation glide might change the type and number of defects; hence, the defect density decreases with plastic deformation. It is worth mentioning that the change rate of dislocation density decreases with increasing initial defects, and the steady-state values of both dislocation and defect densities exist where their rates are close to zero corresponding to Equations (9) and (11) due to the combined effect of multiplication and annihilation of dislocations. The plastic flow resistance increases with increasing initial defect density at $k t / R=0.1$ since defects obstruct the glide dislocations, as shown in Figure 9. The plastic flow resistance increases at the lithiated part $(r>0.8 R)$, which was caused by dislocation activation and multiplication in the case of non-irradiation. However, in the case of $\rho_{f 0}>1.0 \times 10^{-4} \mathrm{~nm}^{-2}$, the plastic flow resistance decreases. Because stress and dislocations contribute to a relatively high annihilation of defects, the effect of annihilation on flow resistance is more than dislocation multiplication. 

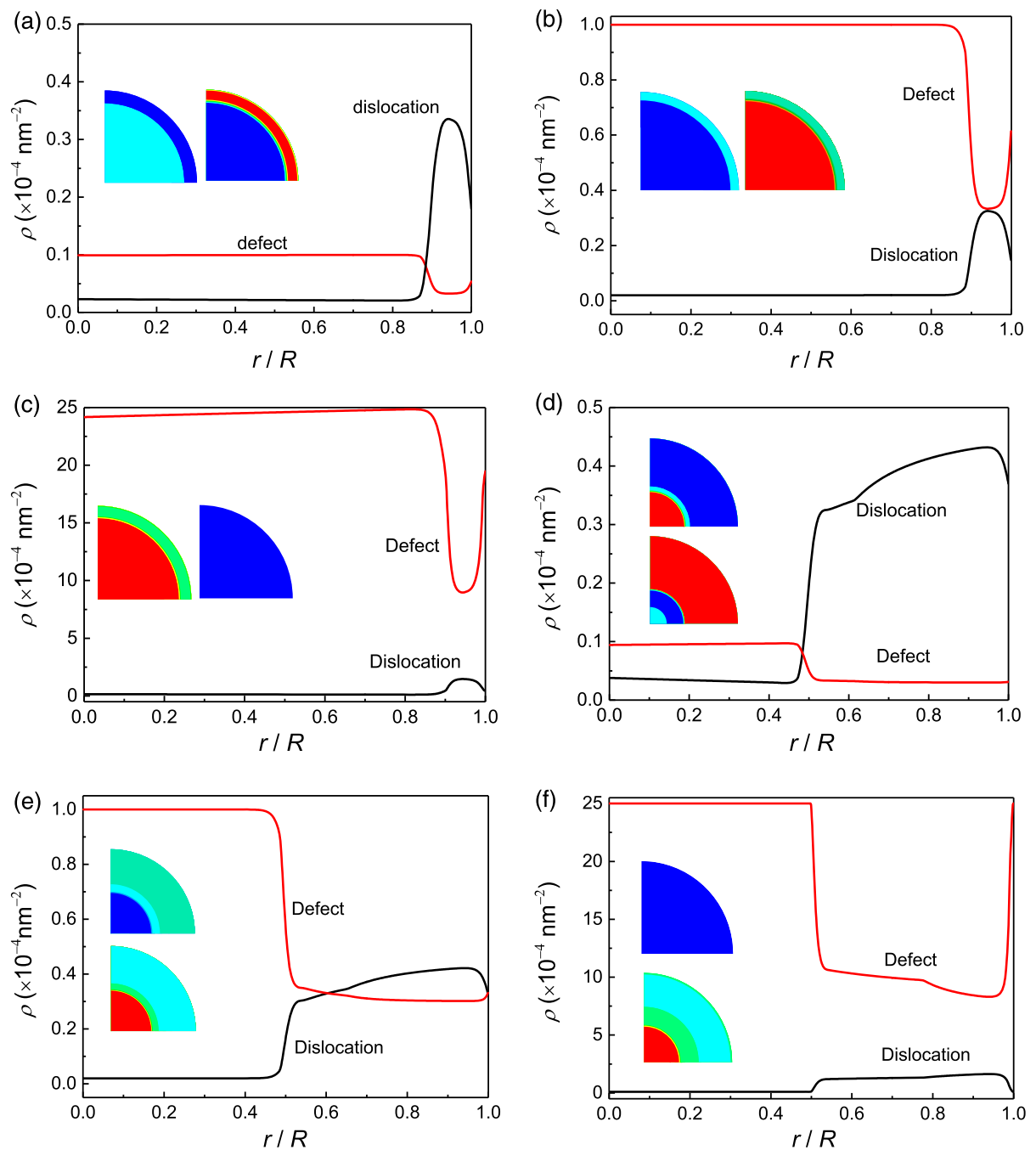

Figure 7. The radial distributions of defect and dislocation densities in a representative irradiation condition that is expressed by the initial defect density during lithiation. (a) $\rho_{\text {fo }}=1.0 \times 10^{-5} \mathrm{~nm}^{-2}, \mathrm{kt} / R=0.1$, (b) $\rho_{f_{\mathrm{o}}}=1.0 \times 10^{-4} \mathrm{~nm}^{-2}, \mathrm{kt} / R=0.1$, and (c) $\rho_{\text {fo }}=5.0 \times 10^{-4} \mathrm{~nm}^{-2}, k t / R=0.1$, for $k t / R=0.5$. The irradiation conditions of (d), (e) and (f) correspond to (a), (b) and (c), respectively.

The stress distributions exhibit a very salient feature due to irradiation and lithiation deformation (see Figure 10). There is a sharp stress change at the phase boundary because of the large lithiation strain and plastic yielding and the hoop stress reverses from initial compression (Figures $10(\mathrm{a}-\mathrm{c})$ ) to tension (Figures $10(b-d)$ ) in the surface layer. Correspondingly, the unlithiated core suffers a tensile hoop stress and then transfers to hydrostatic compression. This is because the hoop stress is accumulated in the surface layer. After irradiation, the trend of stresses distribution is similar to that of non-irradiation. However, in Figures 10(a-b), the irradiation effect is not very evident because 

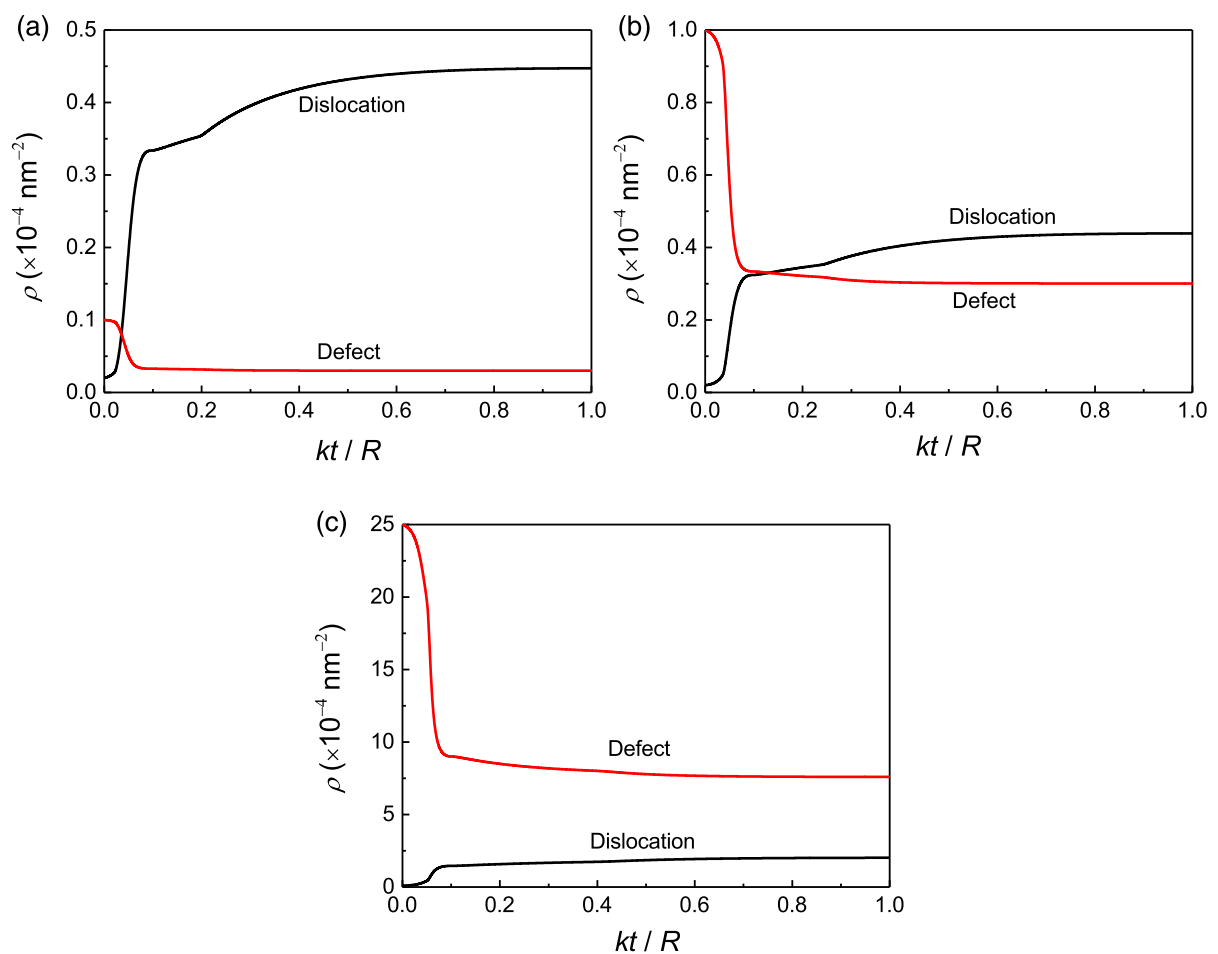

Figure 8. The radical evolution of dislocation and defect densities during lithiation for element A, which is close to the particle surface with different irradiation intensities: (a) $\rho_{f o}=1.0 \times 10^{-5} \mathrm{~nm}^{-2}$, (b) $\rho_{f o}=1.0 \times 10^{-4} \mathrm{~nm}^{-2}$ and (c) $\rho_{f o}=5.0 \times 10^{-4} \mathrm{~nm}^{-2}$.

minority defects play a small part in hindering dislocation slip. As seen in Figures 10(c-d), the hoop stress demonstrates an obvious change in the surface layer with increasing the initial defect density by more stress

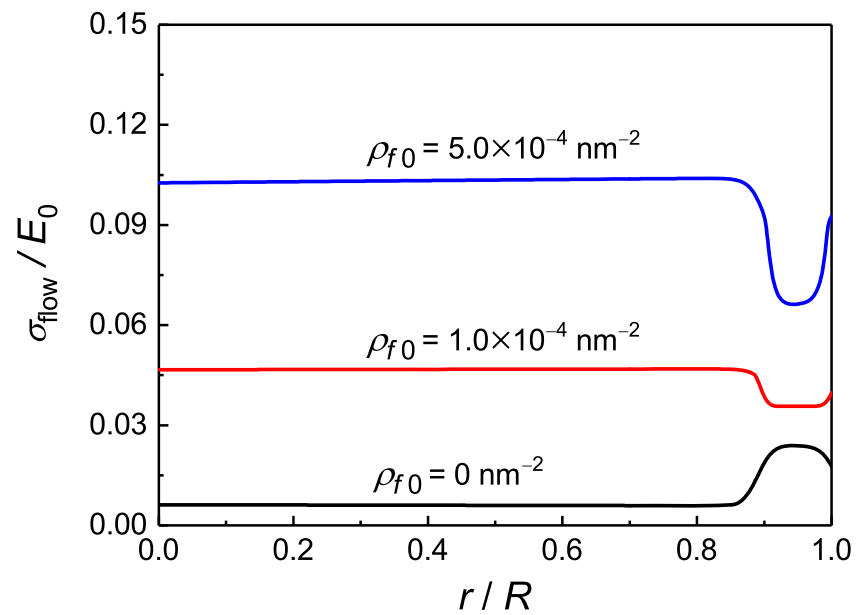

Figure 9. The distribution of yield stress at different initial defect densities and the radial stress distribution is $k t / R=0.1$. 

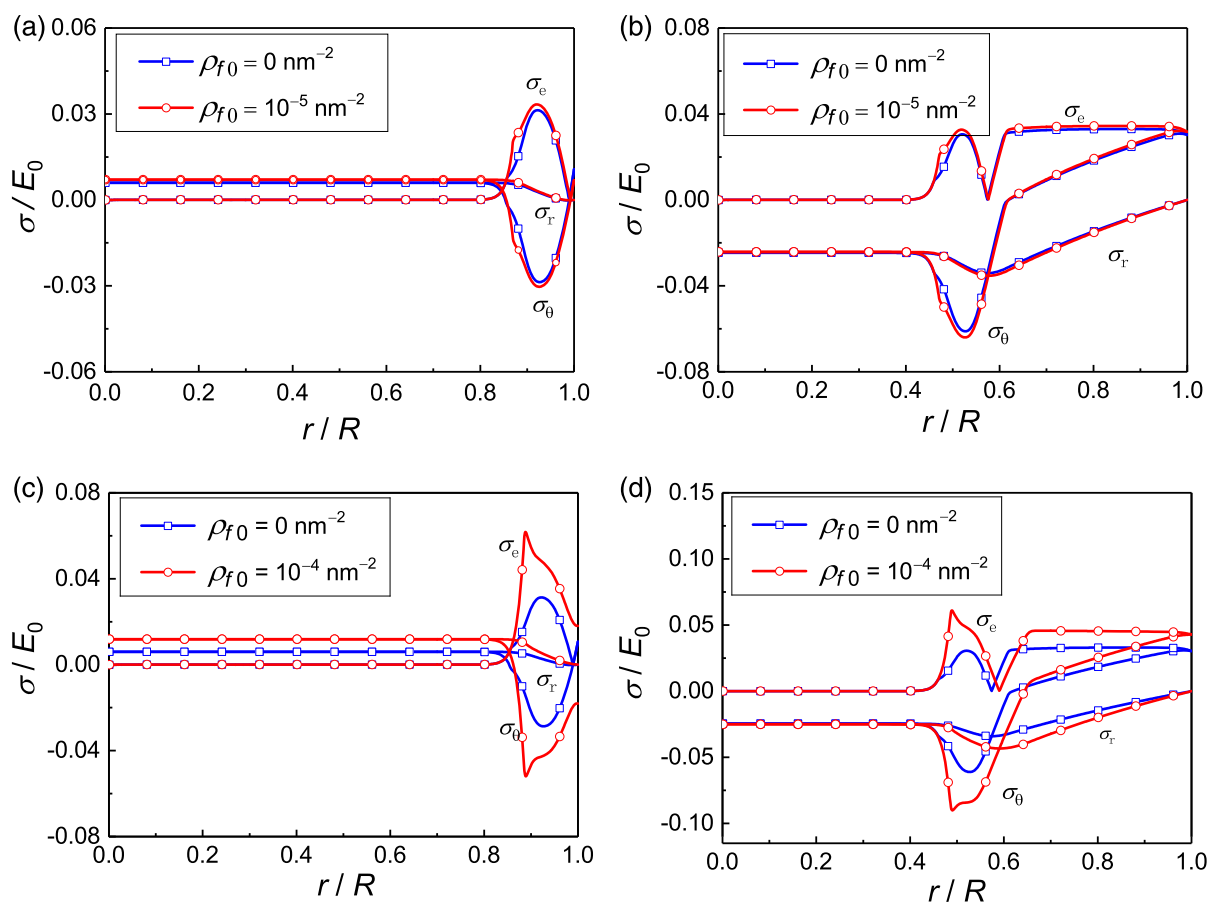

Figure 10. Distributions of radial stress $\sigma_{\mathrm{r}}$, hoop stress $\sigma_{\theta}$ and equivalent stress $\sigma_{\mathrm{e}}$ at different initial defect densities. (a) and (c) are corresponding to $k t / R=0.1$, and (b) and (d) refer to $k t / R=0.5$.

accumulation for the defects distribution to flow stress. Besides, a small sharp peak also appears at the two-phase boundary resulted from the large mismatch stress due to a sharp concentration gradient.

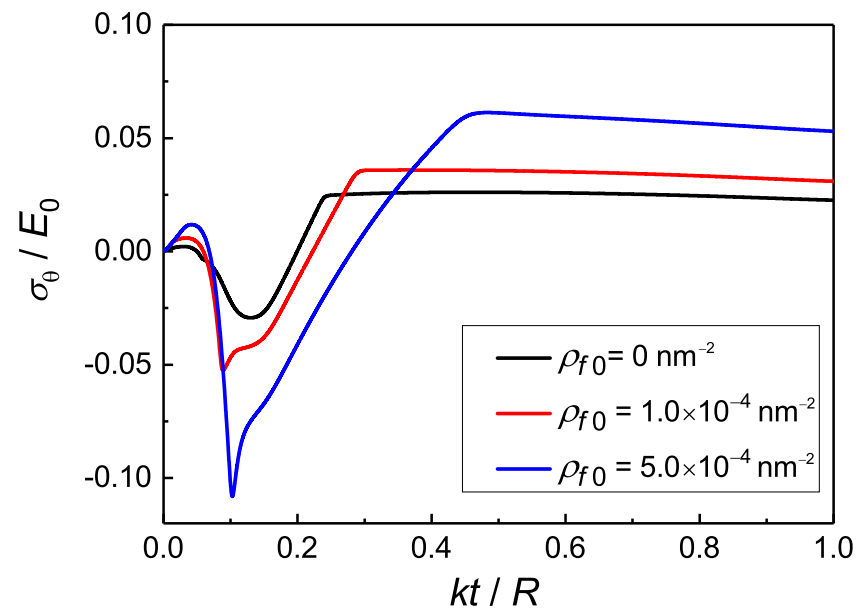

Figure 11. Schematics of the hoop stress experienced by a typical material element A with different initial defect densities. 
To provide a direct physical appreciation of the reversal of hoop compression to tension in the surface layer, we selecting element A (see Figure 3) to probe the effect of irradiation on the hoop stress during Li insertion. As shown in Figure 11, in the unlithiated case, lithiation occurs at the two-phase interface, causing the movement of new reaction along the outward radial direction. This can lead to a radial tensile stress in element A. However, when the reaction front sweeps over the whole element A, local compression stress generates due to the constraint of surrounding materials after a large expansive strain. Finally, the hoop stress reverses from compression to tension since the large lithiation strain and plastic yielding at the front pushes out the material behind it. In the lithiated case, the hoop stress appears a sharp peak when element A is lithiated. As a result, element A experiences compressive elastic unloading, tensile elastic loading and tensile plastic flow. It is worth noting that the tensile plastic flow in the surface layer could cause morphological instability and fracture of electrode particles during $\mathrm{Li}$ insertion. The hoop stress increases with raising the initial defect density at the final stage, which was caused by the impediment of defects to dislocation glides and the increase of plastic flow resistance.

Considering the irradiated-defects on the moving rate of reaction front, Figure 12(a) shows that the defects can improve the concentration-dependent lithiation rate, from the initial $k_{0}=1$ to $k>3$, and then the rate decreases because the interaction between dislocations and defects leads to the vanish of defects during the Li insertion. Furthermore, Figure 12(b) shows the contribution from the stress to the free energy during lithiation. Clearly, it is a linear increase in accordance with the elastic deformation stage; afterwards, $\Delta G_{\text {stress }}$ increases gently due to the plastic flow; and finally, $\Delta G_{\text {stress }}$ shows a huge increase.
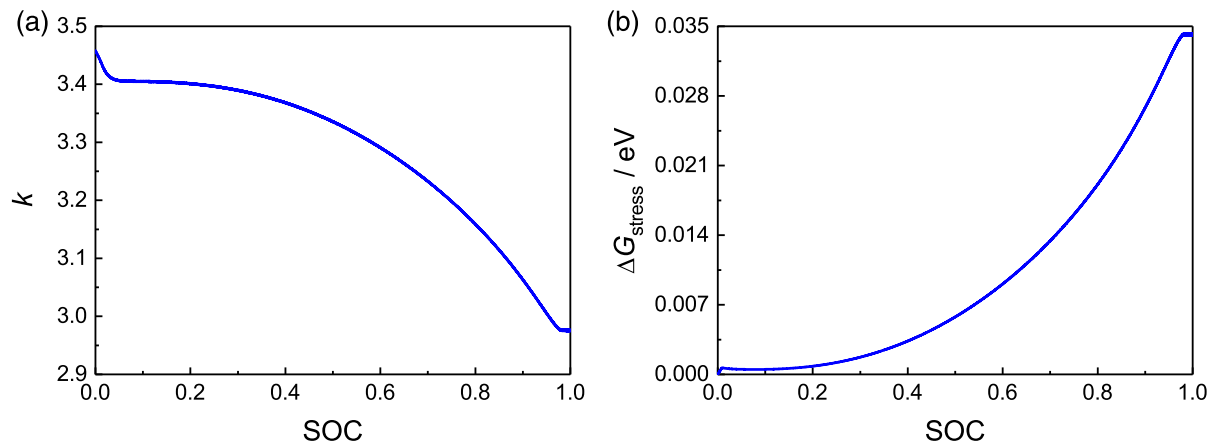

Figure 12. (a) The rate of reaction front and (b) the stress-induced energy barrier for lithiation reaction. 


\section{Conclusions}

In this paper, an electrochemical-irradiated model is developed to explore the interaction between the electrochemical reaction dislocations and irradiation defects on the stress evolution during a two-phase lithiation process. The results indicate that irradiation has an influence on the evolution of stress and the rate of the lithiation reaction front. Especially, when irradiation dose increases during $\mathrm{Li}$ intercalation, the initial yield stress rises owing to irradiation hardening through hindering of defects to dislocations. The plastic flow resistance increases obviously with increasing irradiation dose that is attributed by dislocation multiplication. As irradiation continues and the external load increases to a certain level, defects are obliterated or destroyed, the resistance to dislocation slippage decreases, which leads to a reduction of the external load driving dislocation slip and decrease of plastic flow resistance. Furthermore, the hoop tensile stress in the surface layer of the electrode particle rises as irradiation dose increases, which amplifies morphological instability and implies an easier fracture of electrode particles during $\mathrm{Li}$ insertion. Last but not least, the defects can improve the rate of lithiation reaction front because that the empty space generated by defect facilitates local rearrangement of atoms to readily accept $\mathrm{Li}$ insertion, and the stresses retard lithiation.

However, there are still more factors to be taken into account in this model such as spatial characteristics, temperature and microstructures. It is expected that such a study can be helpful to the understanding of the micro-mechanism of lithiation deformation and applied to study the relationship of mechanical behaviours and electrochemical performance of electrodes in an unconventional environment. We believe that it provides a new way for the development of high-performance LIBs.

\section{Disclosure statement}

No potential conflict of interest was reported by the authors.

\section{Funding}

This work has been supported by the National Natural Science Foundation of China [grant numbers 11872054 and 11772164].

\section{References}

[1] V. Etacheri, R. Marom, R. Elazari, G. Salitra, and D. Aurbach, Challenges in the development of advanced Li-ion batteries: a review, Energ. Environ. Sci. 4 (2011), pp. 3243-3262.

[2] M.R. Palacín and A. de Guibert, Why do batteries fail? Science 351 (2016), pp. 1253292. 
[3] H. Kim, M. Seo, M.-H. Park, and J. Cho, A critical size of silicon nano-anodes for lithium rechargeable batteries, Angew. Chem. Int. Edit. 49 (2010), pp. 2146-2149.

[4] K. Zhao, M. Pharr, J.J. Vlassak, and Z. Suo, Fracture of electrodes in lithium-ion batteries caused by fast charging, J. Appl. Phys. 108 (2010), pp. 073517.

[5] S. Goriparti, E. Miele, F. De Angelis, E. Di Fabrizio, R.P. Zaccaria, and C. Capiglia, Review on recent progress of nanostructured anode materials for Li-ion batteries, J. Power Sources 257 (2014), pp. 421-443.

[6] I. Ryu, J.W. Choi, Y. Cui, and W.D. Nix, Size-dependent fracture of Si nanowire battery anodes, J. Mech. Phys. Solids 59 (2011), pp. 1717-1730.

[7] L.-F. Cui, Y. Yang, C.-M. Hsu, and Y. Cui, Carbon-silicon core-shell nanowires as high capacity electrode for lithium ion batteries, Nano Lett. 9 (2009), pp. 3370-3374.

[8] M. Ebner, F. Marone, M. Stampanoni, and V. Wood, Visualization and quantification of electrochemical and mechanical degradation in Li ion batteries, Science 342 (2013), pp. 716-720.

[9] S. Kim, S.J. Choi, K. Zhao, H. Yang, G. Gobbi, S. Zhang, and J. Li, Electrochemically driven mechanical energy harvesting, Nat. Commun. 7 (2016), pp. 10146.

[10] Y. Wang, Z. Ma, W. Lei, Y. Zou, and C. Lu, Double effect of electrochemical reaction and substrate on hardness in electrodes of lithium-ion batteries, Acta Mech. 227 (2016), pp. 2505-2510.

[11] Z. Xie, Z. Ma, Y. Wang, Y. Zhou, and C. Lu, A kinetic model for diffusion and chemical reaction of silicon anode lithiation in lithium ion batteries, RSC Adv. 6 (2016), pp. 22383-22388.

[12] V.A. Sethuraman, M.J. Chon, M. Shimshak, V. Srinivasan, and P.R. Guduru, In situ measurements of stress evolution in silicon thin films during electrochemical lithiation and delithiation, J. Power Sources 195 (2010), pp. 5062-5066.

[13] S.P.V. Nadimpalli, V.A. Sethuraman, G. Bucci, V. Srinivasan, A.F. Bower, and P.R. Guduru, On plastic deformation and fracture in Si films during electrochemical lithiation/delithiation cycling, J. Electrochem. Soc. 160 (2013), pp. A1885-A1893.

[14] J.Y. Huang, L. Zhong, C.M. Wang, J.P. Sullivan, W. Xu, L.Q. Zhang, S.X. Mao, N.S. Hudak, X.H. Liu, A. Subramanian, et al., In situ observation of the electrochemical lithiation of a single $\mathrm{SnO}_{2}$ nanowire electrode, Science 330 (2010), pp. 1515-1520.

[15] A. Kushima, J.Y. Huang, and J. Li, Quantitative fracture strength and plasticity measurements of lithiated silicon nanowires by in situ TEM tensile experiments, ACS Nano 6 (2012), pp. 9425-9432.

[16] M.T. McDowell, I. Ryu, S.W. Lee, C. Wang, W.D. Nix, and Y. Cui, Studying the kinetics of crystalline silicon nanoparticle lithiation with in situ transmission electron microscopy, Adv. Mater. 24 (2012), pp. 6034-6041.

[17] Y. Wang, Y. Pu, Z. Ma, Y. Pan, and C.Q. Sun, Interfacial adhesion energy of lithium-ion battery electrodes, Extreme Mech. Lett. 9 (2016), pp. 226-236.

[18] P. Zhang, Z. Ma, Y. Wang, Y. Zou, L. Sun, and C. Lu, Lithiation-induced interfacial failure of electrode-collector: A first-principles study, Mater. Chem. Phys. 222 (2019), pp. 193-199.

[19] X.H. Liu, J.W. Wang, S. Huang, F. Fan, X. Huang, Y. Liu, S. Krylyuk, J. Yoo, S.A. Dayeh, A.V. Davydov, et al., In situ atomic-scale imaging of electrochemical lithiation in silicon, Nat. Nanotechnol. 7 (2012), pp. 749-756.

[20] M.T. McDowell, S.W. Lee, W.D. Nix, and Y. Cui, 25th anniversary article: understanding the lithiation of silicon and other alloying anodes for lithium-ion batteries, Adv. Mater. 25 (2013), pp. 4966-4985.

[21] I. Ryu, S.W. Lee, H. Gao, Y. Cui, and W.D. Nix, Microscopic model for fracture of crystalline Si nanopillars during lithiation, J. Power Sources 255 (2014), pp. 274-282. 
[22] X.H. Liu, L. Zhong, S. Huang, S.X. Mao, T. Zhu, and J.Y. Huang, Size-dependent fracture of silicon nanoparticles during lithiation, ACS Nano 6 (2012), pp. 1522-1531.

[23] C. Wang, Z. Ma, Y. Wang, and C. Lu, Failure prediction of high-capacity electrode materials in lithium-ion batteries, J. Electrochem. Soc. 163 (2016), pp. A1157-A1163.

[24] H. Wu, Z. Xie, Y. Wang, C. Lu, and Z. Ma, Modeling diffusion-induced stress on twophase lithiation in lithium-ion batteries, Eur. J. Mech. A-Solid 71 (2018), pp. 320-325.

[25] P. Zuo, and Y. Zhao, Phase field modeling of lithium diffusion, finite deformation, stress evolution and crack propagation in lithium ion battery, Extreme Mech. Lett. 9 (2016), pp. 467-479.

[26] K. Zhao, M. Pharr, Q. Wan, W.L. Wang, E. Kaxiras, J.J. Vlassak, and Z. Suo, Concurrent reaction and plasticity during initial lithiation of crystalline silicon in lithium-ion batteries, J. Electrochem. Soc. 159 (2012), pp. A238-A243.

[27] S. Huang, F. Fan, J. Li, S. Zhang, and T. Zhu, Stress generation during lithiation of highcapacity electrode particles in lithium ion batteries, Acta Mater. 61 (2013), pp. 4354-4364.

[28] B.V. Ratnakumar, M.C. Smart, L.D. Whitcanack, E.D. Davies, K.B. Chin, F. Deligiannis, and S. Surampudi, Behavior of Li-ion cells in high-intensity radiation environments, J. Electrochem. Soc. 151 (2004), pp. A652-A659.

[29] B.V. Ratnakumar, M.C. Smart, A. Kindler, H. Frank, R. Ewell, and S. Surampudi, Lithium batteries for aerospace applications: 2003 Mars exploration Rover, J. Power Sources 119-121 (2003), pp. 906-910.

[30] D.S. Lee, Y.H. Choi, H.D. Jeong, Effect of electron beam irradiation on the capacity fading of hydride-terminated silicon nanocrystal based anode materials for lithium ion batteries. J. Ind. Eng. Chem. 53 (2017), pp. 82-92

[31] M.C. Smart, B.V. Ratnakumar, L.D. Whitcanack, K.B. Chin, S. Surampudi, R. Gitzendanner, F. Puglia, and J. Byers, Lithium-ion batteries for aerospace, IEEE Aero. El. Sys. Mag. 19 (2004), pp. 18-25.

[32] C. Tan, D.J. Lyons, K. Pan, K.Y. Leung, W.C. Chuirazzi, M. Canova, A.C. Co, and L.R. Cao, Radiation effects on the electrode and electrolyte of a lithium-ion battery, J. Power Sources 318 (2016), pp. 242-250.

[33] J. Qiu, D. He, M. Sun, S. Li, C. Wen, J. Hattrick-Simpers, Y.F. Zheng, and L. Cao, Effects of neutron and gamma radiation on lithium-ion batteries, Nucl. Instrum. Meth. B 345 (2015), pp. 27-32.

[34] A. Samin, M. Kurth, and L. Cao, Ab initio study of radiation effects on the $\mathrm{Li}_{4} \mathrm{Ti}_{5} \mathrm{O}_{12}$ electrode used in lithium-ion batteries, AIP Adv. 5 (2015), p. 047110.

[35] Z. Ma, H. Wu, Y. Wang, Y. Pan, and C. Lu, An electrochemical-irradiated plasticity model for metallic electrodes in lithium-ion batteries, Int. J. Plast. 88 (2017), pp. 188-203.

[36] A. Arsenlis, M. Rhee, G. Hommes, R. Cook, and J. Marian, A dislocation dynamics study of the transition from homogeneous to heterogeneous deformation in irradiated bodycentered cubic iron, Acta Mater. 60 (2012), pp. 3748-3757.

[37] N.R. Barton, A. Arsenlis, and J. Marian, A polycrystal plasticity model of strain localization in irradiated iron, J. Mech. Phys. Solids 61 (2013), pp. 341-351.

[38] I.J. Beyerlein and C.N. Tomé, A dislocation-based constitutive law for pure Zr including temperature effects, Int. J. Plast. 24 (2008), pp. 867-895.

[39] X. Xiao, D. Song, J. Xue, H. Chu, and H. Duan, A self-consistent plasticity theory for modeling the thermo-mechanical properties of irradiated FCC metallic polycrystals, J. Mech. Phys. Solids 78 (2015), pp. 1-16.

[40] X. Xiao, D. Song, J. Xue, H. Chu, and H. Duan, A size-dependent tensorial plasticity model for FCC single crystal with irradiation, Int. J. Plast. 65 (2015), pp. 152-167. 
[41] L. Li, H. Wu, and $\mathrm{Z}$. Ma, Neutron radiation on tin anodes of lithium-ion batteries, Radiat. Eff. Defect. S 173 (2018), pp. 1068-1074.

[42] B.N. Singh, A. Horsewell, P. Toft, and D.J. Edwards, Temperature and dose dependencies of microstructure and hardness of neutron irradiated OFHC copper, J. Nucl. Mater. 224 (1995), pp. 131-140.

[43] B.N. Singh, A.J.E. Foreman, and H. Trinkaus, Radiation hardening revisited: role of intracascade clustering, J. Nucl. Mater. 249 (1997), pp. 103-115.

[44] B.N. Singh, D.J. Edwards, and P. Toft, Effect of neutron irradiation and post-irradiation annealing on microstructure and mechanical properties of OFHC-copper, J. Nucl. Mater. 299 (2001), pp. 205-218.

[45] Z. Jiao, and G.S. Was, The role of irradiated microstructure in the localized deformation of austenitic stainless steels, J. Nucl. Mater. 407 (2010), pp. 34-43.

[46] E.H. Lee, T.S. Byun, J.D. Hunn, M.H. Yoo, K. Farrell, and L.K. Mansur, On the origin of deformation microstructures in austenitic stainless steel: part I-microstructures, Acta Mater. 49 (2001), pp. 3269-3276.

[47] E.H. Lee, M.H. Yoo, T.S. Byun, J.D. Hunn, K. Farrell, and L.K. Mansur, On the origin of deformation microstructures in austenitic stainless steel: part II-mechanisms, Acta Mater. 49 (2001), pp. 3277-3287.

[48] M.I. Luppo, C. Bailat, R. Schäublin, and M. Victoria, Tensile properties and microstructure of $590 \mathrm{MeV}$ proton-irradiated pure Fe and a Fe-Cr alloy, J. Nucl. Mater. 283-287 (2000), pp. 483-487.

[49] D. Kiener, P. Hosemann, S.A. Maloy, and A.M. Minor, In situ nano-compression testing of irradiated copper, Nat. Mater. 10 (2011), pp. 608-613.

[50] M. Briceño, J. Kacher, and I.M. Robertson, Dynamics of dislocation interactions with stacking-fault tetrahedra at high temperature, J. Nucl. Mater. 433 (2013), pp. 390-396.

[51] G. Cheng and M. Shehadeh, Multiscale dislocation dynamics analyses of laser shock peening in silicon single crystals, Int. J. Plast. 22 (2006), pp. 2171-2194.

[52] B. Skoczeń, and A. Ustrzycka, Kinetics of evolution of radiation induced micro-damage in ductile materials subjected to time-dependent stresses, Int. J. Plast. 80 (2016), pp. 86-110.

[53] A. Singh, N.R. Tao, M. Dao, and S. Suresh, Repeated frictional sliding properties of copper containing nanoscale twins, Scripta Mater. 66 (2012), pp. 849-853.

[54] Y. Yao, M.T. McDowell, I. Ryu, H. Wu, N. Liu, L. Hu, W.D. Nix, and Y. Cui, Interconnected silicon hollow nanospheres for lithium-ion battery anodes with long cycle life, Nano Lett. 11 (2011), pp. 2949-2954.

[55] K. Zhao, W.L. Wang, J. Gregoire, M. Pharr, Z. Suo, J.J. Vlassak, and E. Kaxiras, Lithium-assisted plastic deformation of silicon electrodes in lithium-ion batteries: a first-principles theoretical study, Nano Lett. 11 (2011), pp. 2962-2967.

[56] Z.-L. Xu, X. Liu, Y. Luo, L. Zhou, and J.-K. Kim, Nanosilicon anodes for high performance rechargeable batteries, Prog. Mater. Sci. 90 (2017), pp. 1-44.

[57] C.K. Chan, H. Peng, G. Liu, K. McIlwrath, X.-F. Zhang, R.A. Huggins, and Y. Cui, Highperformance lithium battery anodes using silicon nanowires, Nat. Nanotechnol. 3 (2008), pp. 31-35.

[58] S.W. Lee, N. Yabuuchi, B.M. Gallant, S. Chen, B.-S. Kim, P.T. Hammond, and Y. ShaoHorn, High-power lithium batteries from functionalized carbon-nanotube electrodes, Nat. Nanotechnol. 5 (2010), pp. 531-537.

[59] Z. Ma, X. Gao, Y. Wang, and C. Lu, Effects of size and concentration on diffusioninduced stress in lithium-ion batteries, J. Appl. Phys. 120 (2016), pp. 025302.

[60] Z. Guo, L. Ji, L. Chen, Analytical solutions and numerical simulations of diffusioninduced stresses and concentration distributions in porous electrodes with particles of different size and shape. J. Mater. Sci. 52 (2017), pp. 13606-13625 
[61] P. Franciosi, and A. Zaoui, Multislip in f.c.c. crystals a theoretical approach compared with experimental data, Acta Metall. 30 (1982), pp. 1627-1637.

[62] K.-S. Cheong, and E.P. Busso, Discrete dislocation density modelling of single phase FCC polycrystal aggregates, Acta Mater. 52 (2004), pp. 5665-5675.

[63] S. Krishna, A. Zamiri, and S. De, Dislocation and defect density-based micromechanical modeling of the mechanical behavior of fcc metals under neutron irradiation, Philos. Mag. 90 (2010), pp. 4013-4025.

[64] H.-J. Lee, J.-H. Shim, and B.D. Wirth, Molecular dynamics simulation of screw dislocation interaction with stacking fault tetrahedron in face-centered cubic $\mathrm{Cu}$, J. Mater. Res. 22 (2007), pp. 2758-2769.

[65] H.-J. Lee, and B. Wirth, Molecular dynamics simulation of the interaction between a mixed dislocation and a stacking fault tetrahedron, Philos. Mag. 89 (2009), pp. 821-841.

[66] J.S. Robach, I.M. Robertson, B.D. Wirth, and A. Arsenlis, In-situ transmission electron microscopy observations and molecular dynamics simulations of dislocation-defect interactions in ion-irradiated copper, Philos. Mag. 83 (2003), pp. 955-967.

[67] Y.N. Osetsky, D. Rodney, and D.J. Bacon, Atomic-scale study of dislocation-stacking fault tetrahedron interactions. part I: mechanisms, Philos. Mag. 86 (2006), pp. 2295-2313.

[68] Z. Ma, Z. Xie, Y. Wang, and C. Lu, Softening by electrochemical reaction-induced dislocations in lithium-ion batteries, Scripta Mater. 127 (2017), pp. 33-36.

[69] K.J. Kim and Y. Qi, Vacancies in Si can improve the concentration-dependent lithiation rate: molecular dynamics studies of lithiation dynamics of Si electrodes, J. Phys. Chem. C 119 (2015), pp. 24265-24275.

[70] A.F. Bower, P.R. Guduru, and V.A. Sethuraman, A finite strain model of stress, diffusion, plastic flow, and electrochemical reactions in a lithium-ion half-cell, J. Mech. Phys. Solids 59 (2011), pp. 804-828.

[71] L. Yu, L. Chen, X. Xiao, Q. Chen, and H. Duan, Constitutive relationship of irradiated metallic materials by Eshelby formalism and micro-mechanical scheme, J. Micromech. Mol. Phys. 1 (2016), pp. 1640006.

[72] R.P. Carreker, and W.R. Hibbard, Tensile deformation of high-purity copper as a function of temperature, strain rate, and grain size, Acta Metall. 1 (1953), pp. 654--663.

[73] A. Arsenlis $\$$, B.D. Wirth, and M. Rhee, Dislocation density-based constitutive model for the mechanical behaviour of irradiated Cu, Philos. Mag. 84 (2004), pp. 3617-3635.

[74] M. Haghi, and L. Anand, A constitutive model for isotropic, porous, elastic-viscoplastic metals, Mech. Mater. 13 (1992), pp. 37-53.

[75] T. Bakos, S.N. Rashkeev, and S.T. Pantelides, $\mathrm{H}_{2} \mathrm{O}$ and $\mathrm{O}_{2}$ molecules in amorphous $\mathrm{SiO}_{2}$ : defect formation and annihilation mechanisms, Phys. Rev. B 69 (2004), pp. 195206. 